\title{
Image-Sensor-Based Fast Industrial-Robot Positioning System for Assembly Implementation
}

\author{
Chyi-Yeu Lin, ${ }^{*}$ Le Thai Son, Yu-Lun Chang, and Ya-Shiun Shiue \\ Department of Mechanical Engineering, National Taiwan University of Science and Technology, \\ 43 Keelung Road, Section 4, Taipei, 10672, Taiwan (Republic of China)
}

(Received October 18, 2016; accepted June 2, 2017)

Keywords: image sensor, object localization, object alignment, robot assembly, autonomous operation

In this paper, an innovative image-technique-based fast industrial-robot positioning system for assembly implementation is presented. Two laser projectors, that is, a laser pointer and a line laser, and an image sensor are used to construct the innovative system to scan and obtain a 3D point cloud model of the object to be assembled. The system is then integrated with a 6-axis industrial robot to define the insertion position in a semi-autonomous manner. This system can save up to $80 \%$ of the manual teaching time for defining robot insertion positions by using a teach pendant. The whole system has been tested on four insertion tasks that require high precision. The results of experiments confirm the effectiveness and efficiency of the system for high-precision tasks. This innovative system can be used in many industrial-robot-based automated assembly tasks.

\section{Introduction}

Industrial robots have been used in many industries for decades. While many tasks can be handled by industrial robots with real-time guidance via different intelligent systems combining sensors and control techniques, the most popular means used for defining the motion (trajectory) of industrial robots for various tasks is the teach pendant. ${ }^{(1-6)}$ The engineer uses the teach pendant to move the end effector of the robot to the required positions with specified orientation in a pointto-point manner. The manual robot teaching job is very time-consuming and it will be effective only if the taught tasks are to be repeatedly performed many times in a long production life cycle, such as in car assembly. Some advanced robots with embedded force control modules can be moved manually, which saves time. However, precise alignment, which requires both position and orientation matching between the part in the gripper of the robot and the workpiece that receives the part is difficult to achieve because of the small tolerance in most assembly tasks of $3 \mathrm{C}$ (Computer, Communication, and Consumer Electronics) products. Therefore, tremendous amounts of time will be used in defining part insertion positions if the traditional teaching method is adopted.

The difficulties of precise alignment comprise two major factors: nonintuitive and changing multi-DOF with efficiency and inaccurate observation and tuning for alignment when viewed manually from the side (not a bird's-eye view along the insertion direction).

In order to reduce the time needed to teach robot insertion positions, we present an innovative vision-technique-based semi-autonomous positioning system for assembly implementation. The

${ }^{*}$ Corresponding author: e-mail: jerrylin@mail.ntust.edu.tw http://dx.doi.org/10.18494/SAM.2017.1637 
proposed system comprises a number of vision subsystems, including an image sensor to capture the background image of the workpiece, a point laser projector and its pairing camera that form a distance measurement device, and a line laser projector and its pairing camera that form a $3 \mathrm{D}$ object scanning device. The proposed semi-autonomous positioning system is used to find the final insertion position of the end effector via a series of vision subsystem operations with the advantage of a reduction in the teaching time by up to $80 \%$.

\section{System Setup}

Figure 1 illustrates the setup of the proposed system in which a Logitech Webcam camera is combined with laser projectors to form a noncontact $3 \mathrm{D}$ measurement system, and the whole system is attached to the end effector of a Denso 6-axis robot. The combination of a gripper and an attached Basler camera is also included in the end effector to conduct semi-autonomous positioning for insertion tasks. The camera installed at the center of the gripper provides visualization to the user. In this system, the task of determining the precise position of the laser segments on the captured image is very important and is the key to obtaining a precise scanned point cloud model. In order to enhance the segmentation of laser rays, a laser filter is used to bypass unwanted light, allowing only laser light of specific wavelengths to reach the camera's image sensor. The filter is fixed in front of the camera entrance, as shown in Fig. 1. Both lasers used in this experiment have a wavelength of $650 \mathrm{~nm}$; therefore, an IR long-pass filter is used to allow only the IR light of 600 $\mathrm{nm}$ wavelength and longer to pass, as can be seen in Fig. 2.

\section{Methods and Analysis}

\subsection{Image sensor calibration}

The camera used in this experiment is the Logitech HD Webcam, which is a pinhole camera. For each pixel in the camera image, there is a line from that pixel that passes through the pinhole. To obtain those lines, we need the intrinsic values of the camera; therefore, we used Zhang's

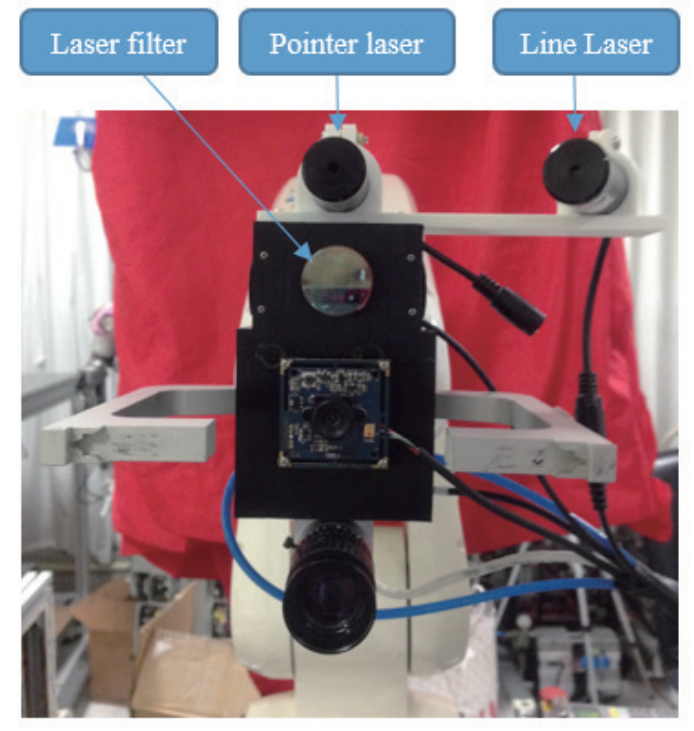

Fig. 1. (Color online) System setup.

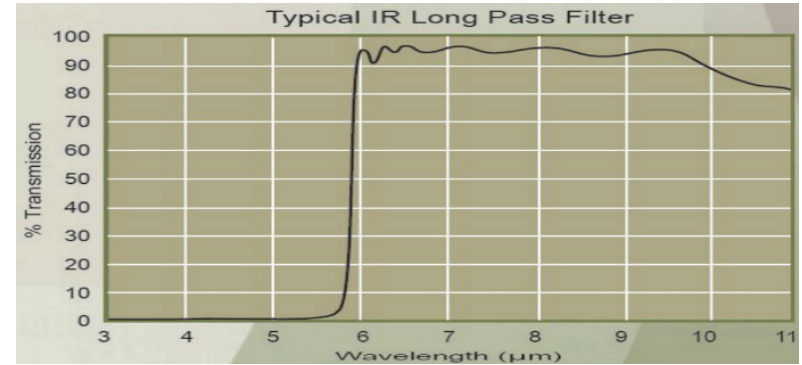

Fig. 2. (Color online) Laser filter specifications. 
method of camera calibration and the backprojection method from OpenCV. Figure 3 shows the calibration process, and the obtained intrinsic values of the camera are listed in Table 1.

The backprojection from the pixel value to the line of $3 \mathrm{D}$ points is then calculated using

$$
s\left[\begin{array}{ccc}
f_{x} & \tau & u_{0} \\
0 & f_{y} & v_{0} \\
0 & 0 & 1
\end{array}\right]^{-1}\left[\begin{array}{c}
u \\
v \\
1
\end{array}\right]=\left[\begin{array}{c}
X \\
Y \\
Z
\end{array}\right]
$$

Since the intrinsic values of the camera sensor are constant, for every single pixel $(u, v)$, we can find a line of infinite 3D points where the position of each point is determined by the value of scale factors.

\subsection{Hand-eye calibration}

Since the camera is fixed on the robot end effector, the camera calibration results can be used to obtain the transformation from the camera coordinate to the robot's end-effector coordinates or the robot's base coordinates. ${ }^{(7)}$ In this hand-eye calibration, the calibration board is fixed at one position to act as the world coordinates. The same calibrating process is applied to 13 different camera positions in the world coordinates. By pairing these camera positions and orientations in the world coordinates with their corresponding end-effector positions and orientations in the robot's base coordinates, we can generate the transformation. Figure 4 shows the calibration process. The camera position and orientation in the end-effector coordinate are listed in Table 2.

The transformation matrix shown in Eq. (2), used to transform a 3D point from the camera's coordinates to the end-effector's coordinates, is constant and can be derived from values in Table 2.

$$
{ }^{E} T_{c}=\left[\begin{array}{cccc}
-0.03834 & 0.9992 & 0.0003 & -46.2089 \\
-0.9991 & -0.0383 & -0.0175 & 1.3848 \\
-0.0175 & -0.001 & 0.9998 & 93.6309 \\
0 & 0 & 0 & 1
\end{array}\right]
$$

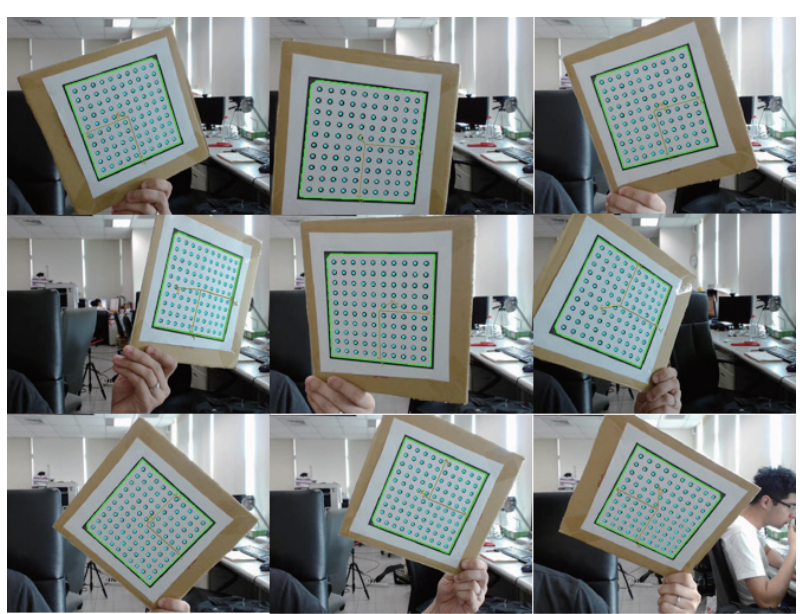

Fig. 3. (Color online) Camera calibration process.
Table 1

Camera intrinsic values.

\begin{tabular}{lc}
\hline Intrinsic parameter & Value \\
\hline Focus length $(f)$ & $5.07625 \mathrm{~mm}$ \\
Skew coefficient $(\tau)$ & 0 \\
Scale factor in $x$-axis $\left(m_{x}\right)$ & $8.3 \mathrm{~nm}$ \\
Scale factor in $y$-axis $\left(m_{y}\right)$ & $8.3 \mathrm{~nm}$ \\
Principal point in $x$-axis $\left(u_{0}\right)$ & 314.962 \\
Principal point in $y$-axis $\left(v_{0}\right)$ & 232.344 \\
\hline
\end{tabular}




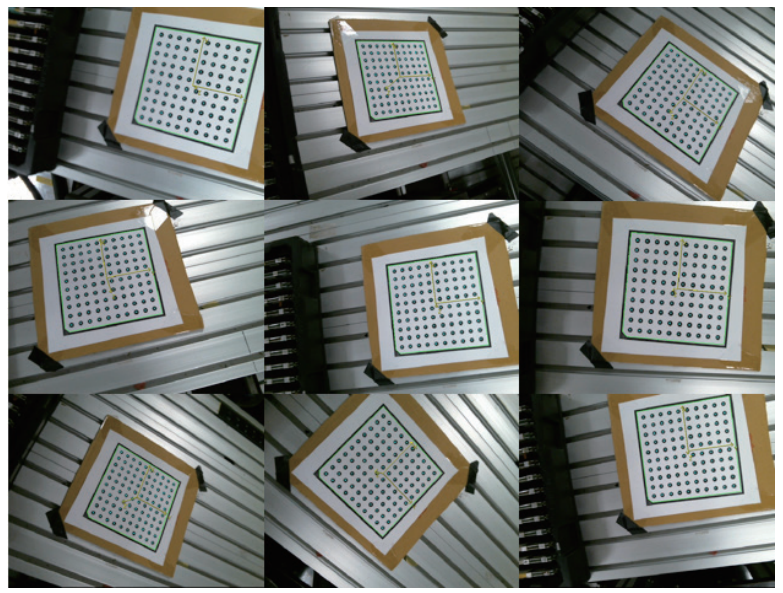

Fig. 4. (Color online) Hand-eye calibration process.
Table 2

Camera in tool pose.

\begin{tabular}{lc}
\hline Camera in tool coordinates & Value \\
\hline Translation on $x$-axis & $-46.2089 \mathrm{~mm}$ \\
Translation on $y$-axis & $1.38484 \mathrm{~mm}$ \\
Translation on $z$-axis & $93.631 \mathrm{~mm}$ \\
Rotation on $x$-axis & $359.941^{\circ}$ \\
Rotation on $y$-axis & $1.00457^{\circ}$ \\
Rotation on $z$-axis & $267.802^{\circ}$ \\
\hline
\end{tabular}

\subsection{Laser segmentation}

The image obtained by the camera with the laser filter must undergo the segmentation process to separate the laser pixels from the background. This process is done by the adaptive threshold method from OpenCV, in which pixels are classified as either "dark" or "light". The after-threshold image of a line laser will have lines wider than one pixel. Since a single-pixel line is needed to calculate the laser's 3D position, we implemented the thinning operation to reduce lines to be one pixel wide. In an image sensor frame, the thinning operation uses a 3 by 3 structure element matrix to remove the outer pixel of the line laser, and the process is applied repeatedly until a single pixel line remains. Figure 5 shows a frame with sequential processes from the threshold method to thinning. The image after thinning is used to detect lines by the detection method from OpenCV. ${ }^{(8)}$

\subsection{D Measurement with laser pointer}

Figure 6 shows the concept of using a laser pointer to transform a 2D point to a 3D point with distance information. Since the camera and laser pointer are fixed with a distance $\mathrm{h}$ between their centerlines, the distance $d$ from the laser projector to its projection point on the object surface can be determined by the pixel value of the pointer on the image. After receiving the pixel value of the pointer projection using the above laser segmentation method, we can easily compute the distance $d$ :

$$
d=\frac{h}{\tan \theta}=\frac{h * f}{p f c * m_{z}} .
$$

The 3D coordinates of the laser projection are defined by $x=0, y=0$, and $z=D$; therefore, a transformation from the laser coordinates to the robot's base coordinates is applied to the point $\mathrm{P}(x, y, z)$ $=(0,0, d)$ to obtain the final $3 \mathrm{D}$ point with respect to the robot's base $P_{B}$. Equation (4) shows the transformation where $(\phi, \theta, \psi)$ is the rotation vector for transformation from the base coordinates to the end-effector coordinates. ${ }^{(9)}$ 

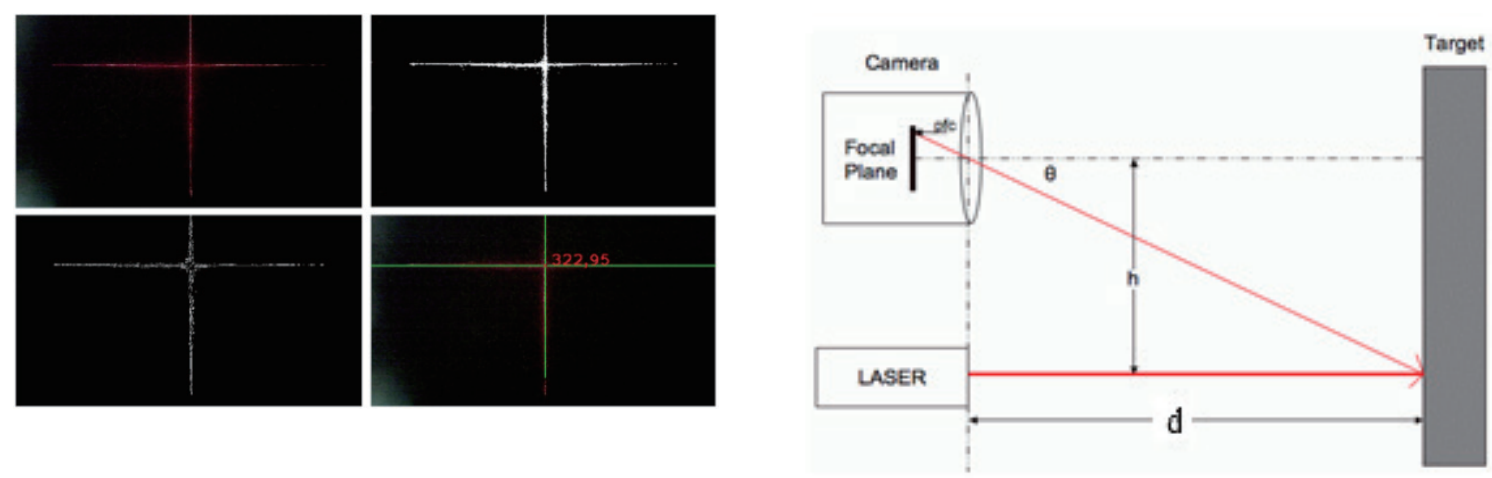

Fig. 5. (Color online) Laser segmentation after applying an adaptive threshold (upper right) and the

Fig. 6. (Color online) Range finder system. thinning process (lower left).

$$
P_{B}=\left[\begin{array}{cccc}
c \phi c \theta & c \phi s \theta s \psi-s \phi c \psi & c \phi s \theta c \psi+s \phi s \psi & { }^{x} R \\
s \phi c \theta & s \phi s \theta s \psi+c \phi c \psi & s \phi s \theta c \psi-c \phi s \psi & { }^{y} R \\
-s \theta & c \theta s \psi & c \theta c \psi & { }^{z} R \\
0 & 0 & 0 & 1
\end{array}\right]\left[\begin{array}{cccc}
1 & 0 & 0 & 2 B \\
0 & 1 & 0 & 0 \\
0 & 0 & 1 & 0 \\
0 & 0 & 0 & 1
\end{array}\right]\left[\begin{array}{c}
0 \\
0 \\
d \\
1
\end{array}\right]
$$

The precisions of $x$ and $y$ only depend on the robot arm precision. In this study, we used the Denso 6-axis robot arm with a precision of $\pm 0.2 \mathrm{~mm}$. The depth precision is shown in Fig. 7 and Table 3.

\subsection{D scanner with line laser}

The line laser projects a plane of light that intersects the object surface and forms projection points. As shown in Fig. 8, since we fixed the laser and the camera on the robot's end effector, the laser plane is also fixed on the camera coordinates. To calculate the laser plane equation, we used 2 calibration boards to project the line laser and then the backprojection method from the camera calibration to find the 3D positions of intersection points between the laser and calibration boards, which correspond to the camera coordinates. Three randomly selected points are used to calculate the plane equation. Figure 9 shows an example of obtaining a laser plane. ${ }^{(10)}$

After obtaining the plane equation, we applied Eq. (1) to every single pixel of the laser projection (shown in red) on the image to obtain the array of light. The 3D position of the laser projection on the object will be the intersection between the laser plane and the light array (see Fig. 9).

The final 3D position of a point belonging to the laser projection corresponding to the camera coordinates can be derived by the following equation.

$$
\begin{gathered}
X=x^{\prime} Z / f_{x} \\
Y=y^{\prime} Z / f_{y} \\
Z=\frac{-D}{A x^{\prime} / f_{x}+B y^{\prime} / f_{y}+C}
\end{gathered}
$$




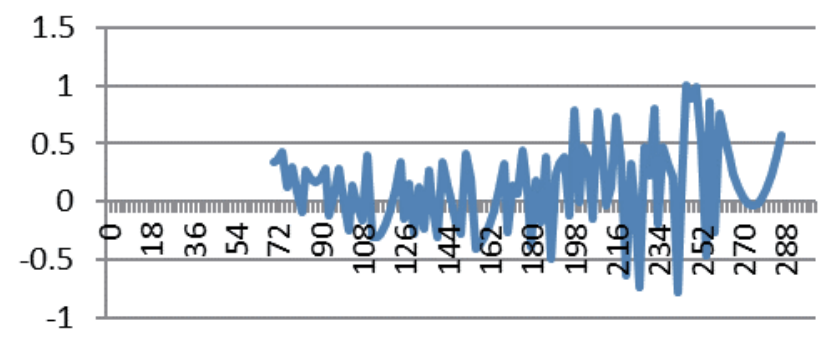

Fig. 7. (Color online) Depth precision test.

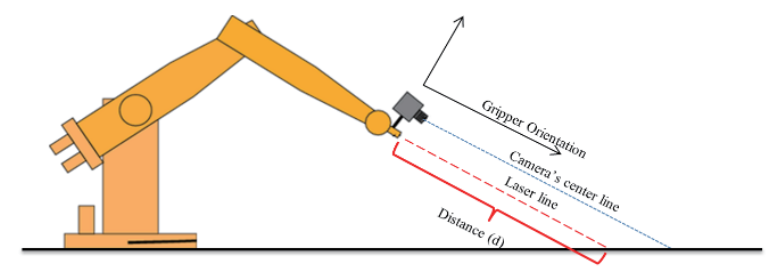

Fig. 8. (Color online) Integrating 3D measurement system.
Table 3

Depth precision of the system.

\begin{tabular}{lc}
\hline Distance range & Depth precision \\
\hline $70-200 \mathrm{~mm}$ & $\pm 0.5 \mathrm{~mm}$ \\
$200-290 \mathrm{~mm}$ & $\pm 1.0 \mathrm{~mm}$ \\
\hline
\end{tabular}

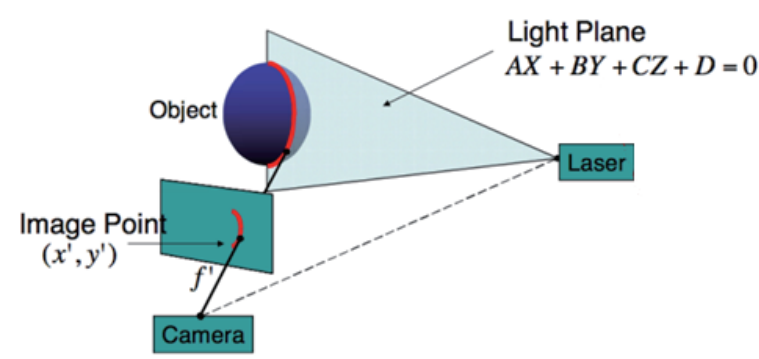

Fig. 9. (Color online) Laser plane in the camera coordinates.

To verify the result of the scanner, we measured the real size of the scanned object and compared it with the scanned result. Figure 10 shows scanned results of the model object and the corresponding error.

Using the hand-eye calibration result, the obtained 3D points can now be transformed from the camera coordinates to the robot's base coordinates with the following equation.

$$
\begin{aligned}
P_{B} & =\left[\begin{array}{cccc}
c \phi c \theta & c \phi s \theta s \psi-s \phi c \psi & c \phi s \theta c \psi+s \phi s \psi & { }^{x} R \\
s \phi c \theta & s \phi s \theta s \psi+c \phi c \psi & s \phi s \theta c \psi-c \phi s \psi & { }^{2} R \\
-s \theta & c \theta s \psi & c \theta c \psi & { }^{z} \\
0 & 0 & 0 & 1
\end{array}\right] \\
& {\left[\begin{array}{cccc}
-0.03834 & 0.9992 & 0.0003 & -46.2089 \\
-0.9991 & -0.0383 & -0.0175 & 1.3848 \\
-0.0175 & -0.001 & 0.9998 & 93.6309 \\
0 & 0 & 0 & 1
\end{array}\right]\left[\begin{array}{c}
X_{C} \\
Y_{C} \\
Z_{C} \\
1
\end{array}\right] }
\end{aligned}
$$

Figure 11 shows some scanning results corresponding to the robot's base coordinates.

\section{Implementation}

In order to test the robustness and reliability of the developed system for industrial robot tasks, we conducted four parts insertion experiments in four different objects. These tasks all require high precision of $0.25-0.5 \mathrm{~mm}$ in position. 


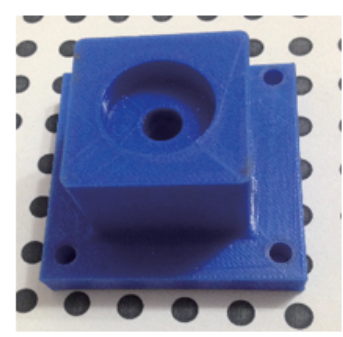

Model object

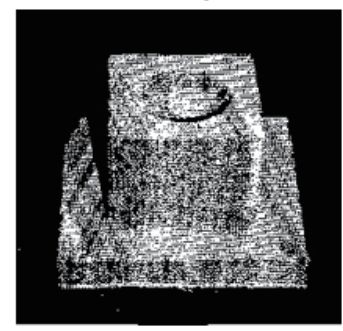

Error $=0.1816 \mathrm{~mm}$

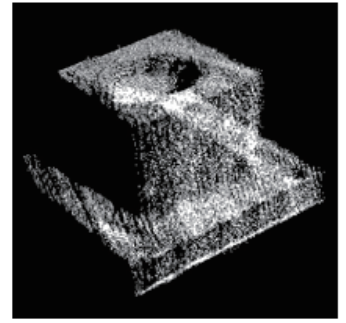

Error $=0.4155 \mathrm{~mm}$

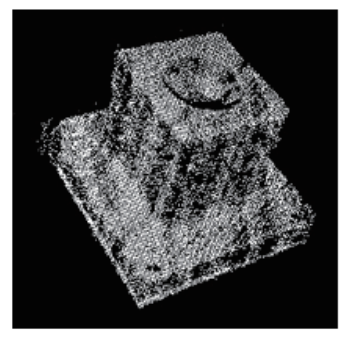

Error $=0.3174 \mathrm{~mm}$

Fig. 10. (Color online) Scanned results and errors.
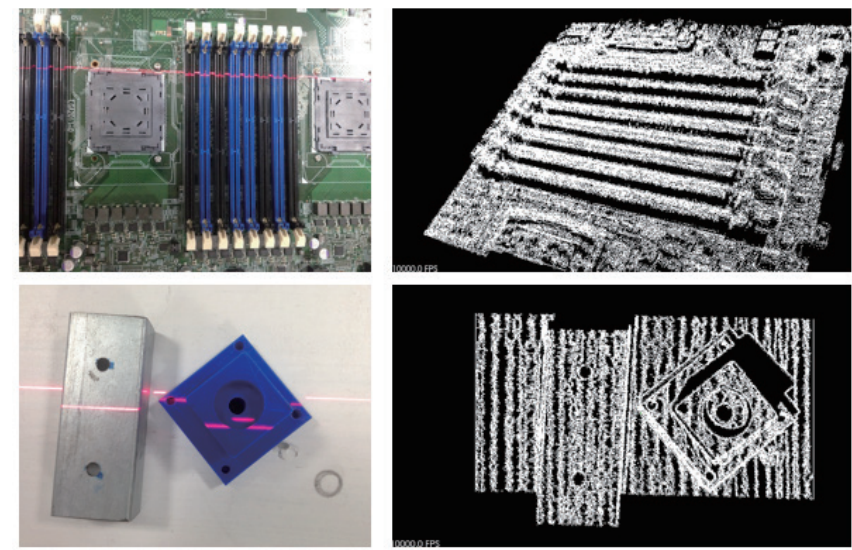

Fig. 11. (Color online) Scanned objects in the robot's base coordinates.

\subsection{Dual in-line memory module (DIMM) insertion}

DIMM insertion requires the industrial robot to insert DIMM into a DIMM socket in a circuit board, randomly placed on the work space. First of all, the laser pointer is used to locate some points on the circuit board surface, to calculate the normal vector of this surface, and then to guide the robot's end effector to the desired position. The line laser then scans through the board to locate the DIMM positions as well as determine the DIMM height. The shape-based matching (SBM) method from Halcon ${ }^{(11)}$ is then applied to precisely match the DIMM heads and locate the final insertion position from the scanned point cloud, as shown in Fig. 12.

The result showed a successful implementation of a system for a socket insertion task. The system can deal with the high precision application with a position error of $\pm 0.5 \mathrm{~mm}$. The GUI system can be seen in Fig. 13.

\subsection{Dot matrix LED insertion}

This task requires the insertion of a dot matrix LED into an 8051MCU board, as shown in Fig. 14. Line laser scanning generated the point cloud model, as shown in Fig. 15. The back of the dot LED has two rows of 12 pins. The middle point is located at the center between the 6th and 7th pins. Therefore, to determine the insertion position, two pins on each side are selected. The SBM method is then applied to precisely match the pair of circular pinholes and locate the final insertion position from the scanned point cloud, as shown in Fig. 16.

\subsection{Clock oscillation insertion}

This task is the insertion of a 4-pin clock oscillator into a motor control board, both of which are shown in Fig. 17. The line laser scanning and the point cloud model are shown in Fig. 18. The 

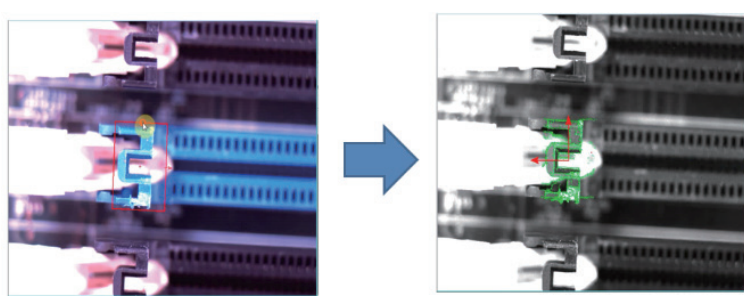

Fig. 12. (Color online) Halcon SBM.

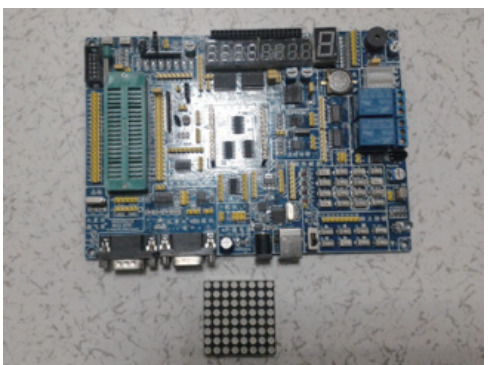

Fig. 14. (Color online) Dot matrix LED and 8051MCU board.

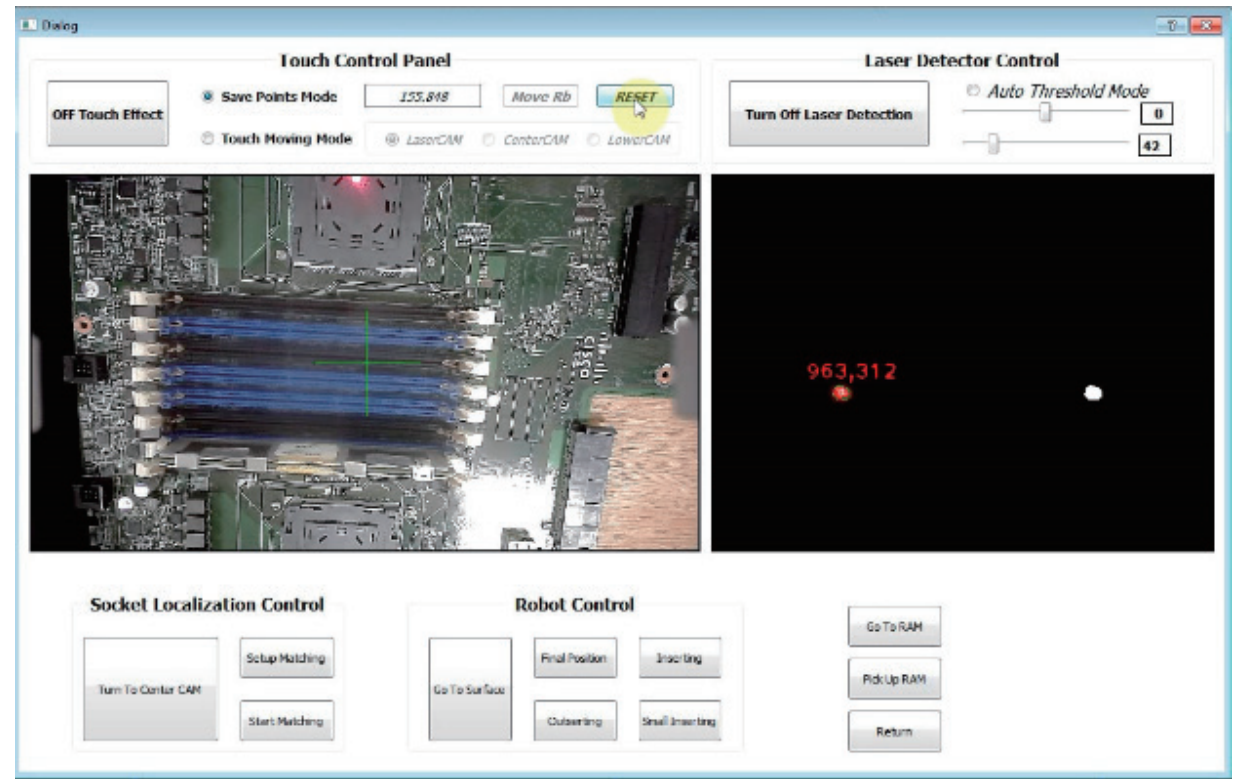

Fig. 13. (Color online) Socket-insertion GUI system.

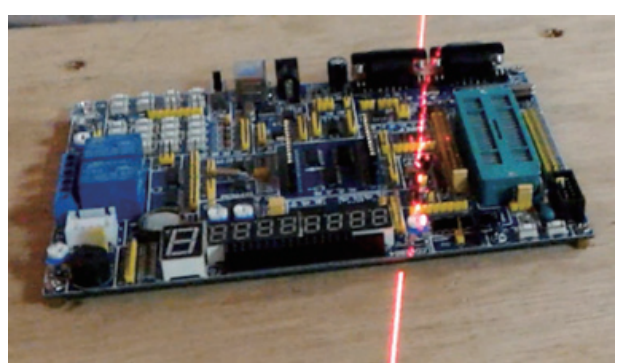

(a)

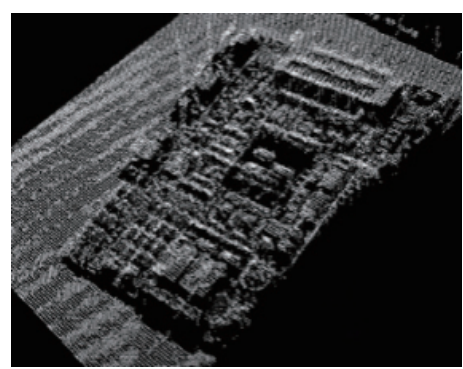

(b)

Fig. 15. (Color online) Line laser scanning and the point cloud model.

clock oscillator socket has 4 pins located at the 4 symmetric corners, as shown in Fig. 19. The two rows of pins on the top and the bottom are separated by a distance of $7.62 \mathrm{~mm}$. To determine the central insertion position, the middle positions of the two upper corner pins are obtained by the SBM method. After that, this middle position is shifted $3.81 \mathrm{~mm}(7.62 \mathrm{~mm} / 2)$ to generate the final insertion position, as shown in Fig. 20. 

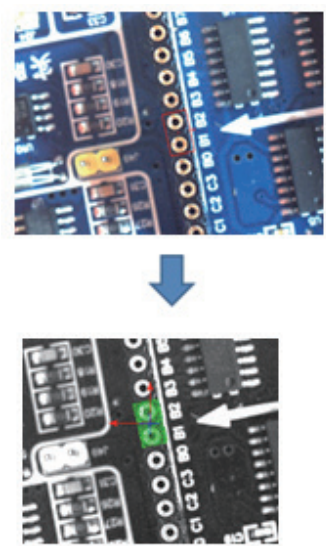

Fig. 16. (Color online) Halcon SBM.

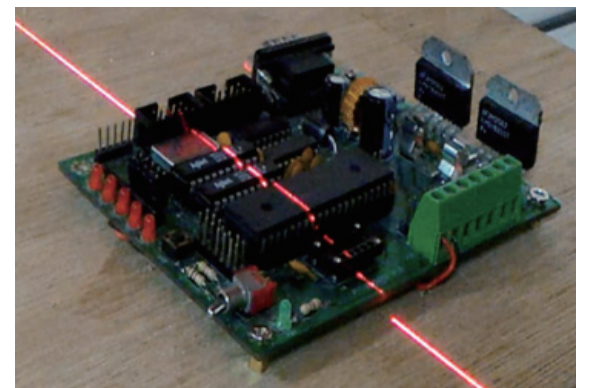

(a)

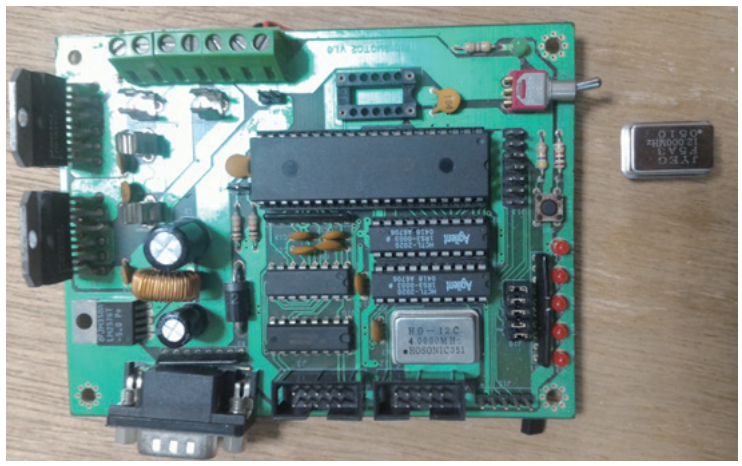

Fig. 17. (Color online) Clock oscillator and motor control board.

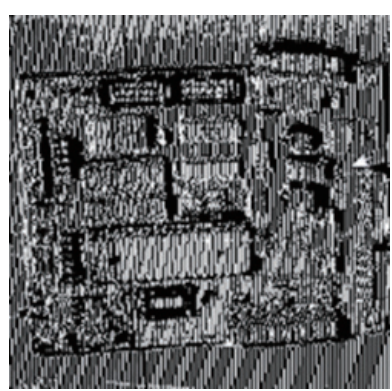

(b)

Fig. 18. (Color online) Line laser scanning and the point cloud model.

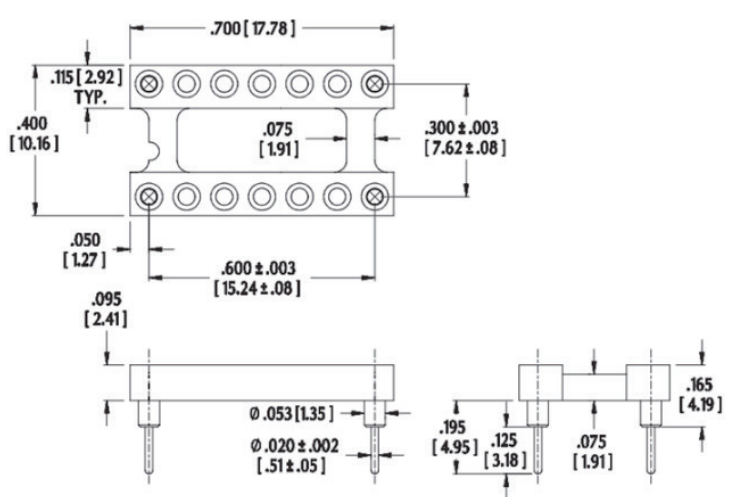

Fig. 19. 4-pin clock oscillator socket.
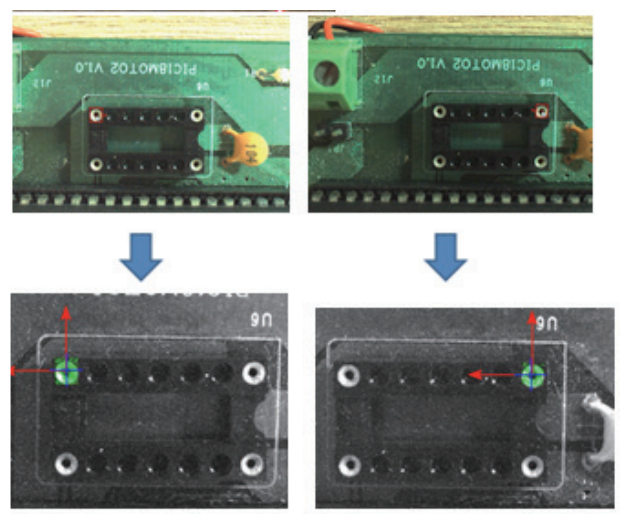

Fig. 20. (Color online) Halcon SBM.

\subsection{Flash IC insertion}

The fourth and final insertion implementation is the insertion of a CMOS flash memory IC to the PCBA of a personal computer, as shown in Fig. 21. The line scanning and the generated point cloud model are shown in Fig. 22. The flash memory has 2 rows of 16 pins in each side of the IC, separated by a distance of $15.24 \mathrm{~mm}$, as shown in Fig. 23. Similar to the clock oscillator insertion 
implementation, the middle position of the top row of pins is calculated by the SBM method. After that, this middle position is shifted $7.62 \mathrm{~mm}(15.24 \mathrm{~mm} / 2)$ to generate the final insertion position, as shown in Fig. 24. The GUI system is shown in Fig. 25.

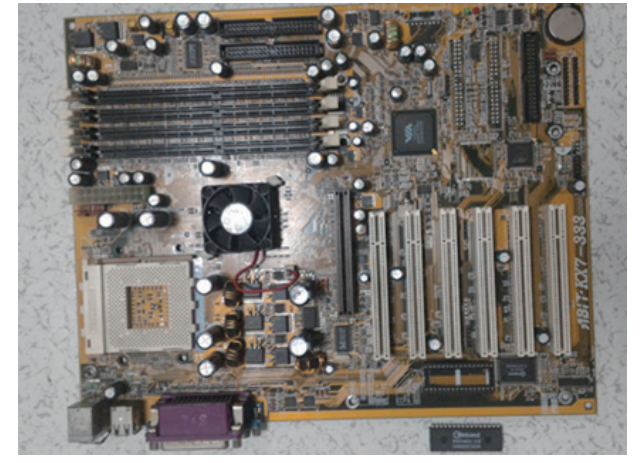

Fig. 21. (Color online) PCBA and CMOS flash memory IC.

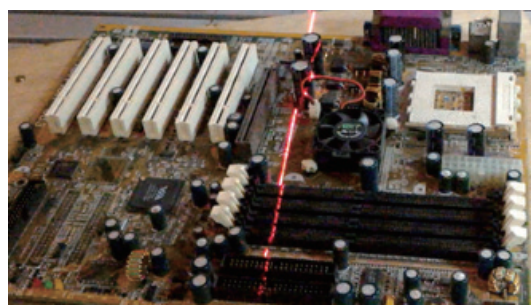

(a)

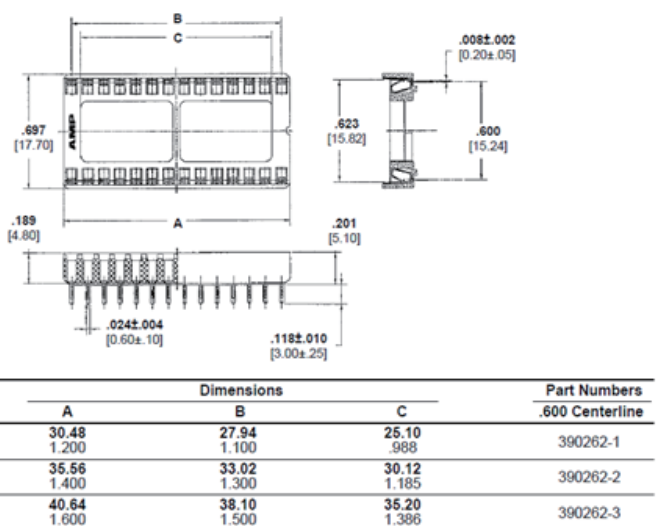

Fig. 23. CAD file of the flash memory IC socket.

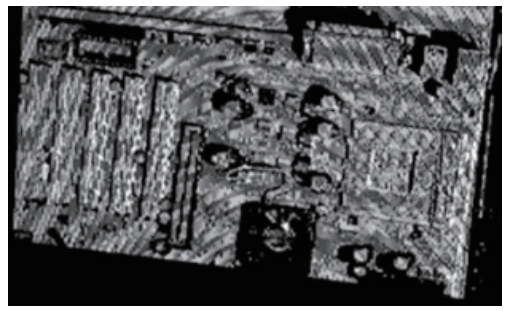

(b)

Fig. 22. (Color online) Line laser scanning and the point cloud model.
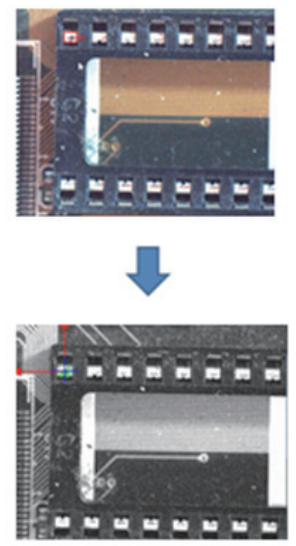

Fig. 24. (Color online) Halcon SBM.

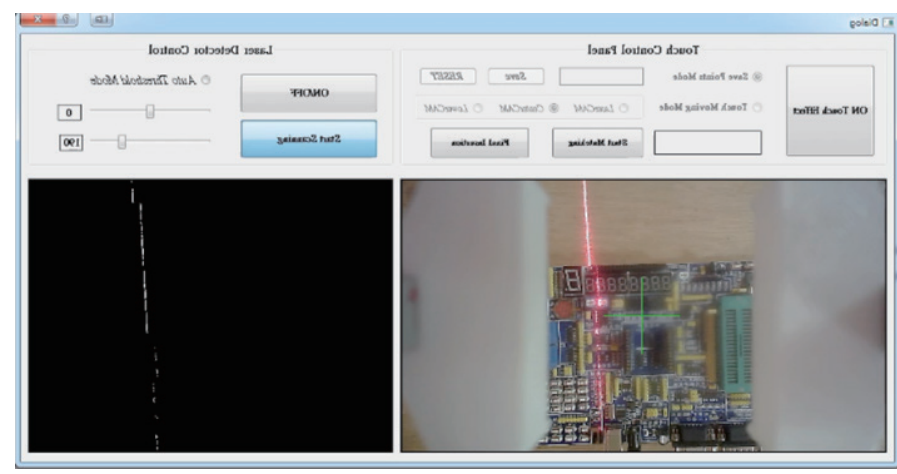

Fig. 25. (Color online) Flash IC insertion GUI system. 


\section{Conclusions}

We presented a noncontact 3D measurement system comprising an image sensor combined with a line laser projector, and the system was integrated with a 6-axis industrial robot. The system can be used to reduce the manual robot teaching time by up to $80 \%$ by using the image-techniqueenhanced autonomous positioning system. The results from experiments on 4 insertion tasks indicated that the proposed system can successfully work in assembly tasks with the tolerance level of $\pm 0.5 \mathrm{~mm}$, which satisfies the requirement of most insertion tasks of most $3 \mathrm{C}$ products. The system error may become larger when the laser segmentation is affected by undesirable light exposure or light reflection. The system used half of the maximum image sensor resolution since our computing system cannot catch up to the maximum load associated with the maximum resolution. In future work, we aim to improve the computing system and further raise the ability of laser segmentation to gain higher precision and stable point cloud models.

\section{Acknowledgments}

This research was conducted with the support of the Ministry of Science and Technology, Taiwan (R.O.C.) through grant number 104-2221-E-011-059-MY3 at the Department of Mechanical Engineering, National Taiwan University of Science and Technology.

\section{References}

1 R. Van Ham, T. G. Sugar, B. Vanderborght, K. W. Hollander, and D. Lefeber: IEEE Rob. Autom. Mag. 16 (2009) 3.

2 S. Tottori, L. Zhang, F. Qiu, K. K. Krawczyk, A. Franco-Obregón, and B. J. Nelson: Adv. Mater. 24 (2012) 811.

3 W. Zesch, M. Brunner, and A. Weber: IEEE Proc. Robotics and Automation (1997) p. 2.

4 E. J. Van Henten, J. Hemming, B. A. J. van Tuijl, J. G. Kornet, J. Meuleman, J. Bontsema, and E. A. van Os: Auton. Rob. 13 (2002) 241.

5 R. A. Brooks: Int. J. Rob. Res. 2 (1983) 19.

6 A. Liu, G. Tharp, L. French, S. Lai, and L. Stark: IEEE Trans. Rob. Autom. 9 (1993) 638.

7 K. Daniilidis: Int. J. Rob. Res. 18 (1999) 286.

8 Camera calibration OpenCV: http://docs.opencv.org/2.4/doc/tutorials/calib3d/camera_calibration/camera_ calibration.html

9 D. Lopez-Escogido and A. De Luca: Proc. 9th Int. Conf. Electrical Engineering, Computing Science and Automatic Control (CCE, 2012) pp. 1-6.

10 R. Marani, M. Nitti, G. Cicirelli, T. D’Orazio, and E. Stella: Adv. Mech. Eng. 2013 (2013).

11 Halcon Shape-Based Matching: http://download.mvtec.com/halcon-9.0-solution-guide-ii-b-shape-basedmatching.pdf 